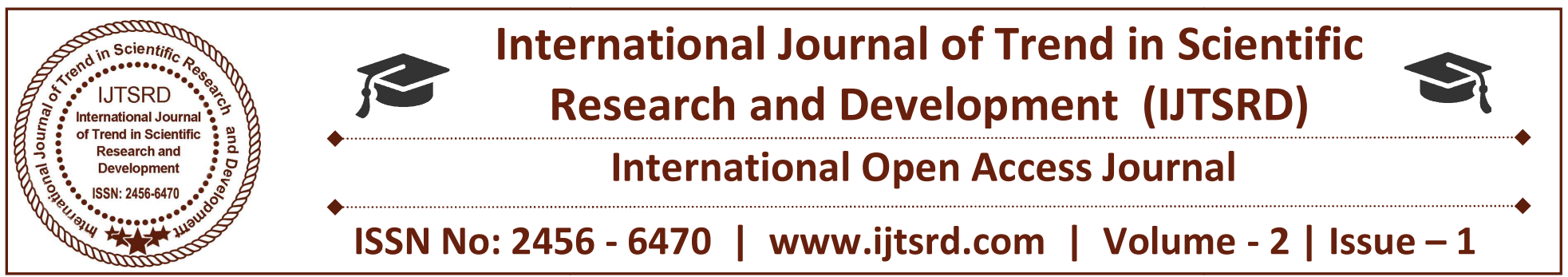

\title{
Traffic Light Controller for the Congested Urban Route using PLC \& Ambulance Detection using RF Transmitter \& Receiver with Lab VIEW
}

Mamatha R

M.E Student, Control \&Instrumentation, Department of Electrical Engineering,University Visveshwaraya College of Engineering, K.R.Circle, Bengaluru-560001, India

\author{
Dr. H.Prasanna Kumar \\ Assistant Professor, Control \& Instrumentation, \\ Department of Electrical Engineering, University \\ visveshwaraya College of Engineering, K.R.Circle, \\ Bengaluru-560001, India
}

\section{ABSTRACT}

Traffic signals are the most convenient method of controlling traffic in a busy junction. Present traffic Signals fail to control the traffic effectively when a particular lane has got more traffic than the other lanes. In this paperan intelligent traffic control system using PLC is proposed. System measures the traffic density on each road bycounting the number of vehicles and then takes the decision. Programming is done using ladder diagram. Seimens S7-1200 PLC is have been used for the system implementation. Traffic management on the road has become a severe problem of today's society because of growth of the urbanization. This leads to traffic jam at the traffic junctions which in turn causes delay to ambulances. In order to overcome this problem, this paper presents a simple ambulance controlled traffic system. The main objective of this system is that to control the traffic, allowing an ambulance to arrive at a particular location without it having to stop anywhere until the destination is reached. This system includes RF Transmitter \& Receiver technology and Lab VIEW software.

Keywords: Traffic signal, PLC, Sensors, Ambulance, RF Transmitter \& Receiver, Lab VIEW

\section{INTRODUCTION.}

Nowadays, vehicles have rapidly increasing throughout the world, particularly in large urban areas. Therefore the need arises for a simulating and

optimizing system for the traffic controllers to better accommodate this increasing demand by road users around the world. Traffic lights are commonly used device to regulate roadway intersection traffic with a view to both safety and smoothness of vehicle flow. Even though traffic lights are known as the best device in controlling traffic flow for road users, yet accidents reported at the traffic junction is very common.

There are 2 methods in controlling the traffic light system placed on a certain junction. The most common one is the sequencing method, whereby the traffic light system is designed to operate according to the preprogrammed sequence without any consideration of real time behavior. The second method is the demand based controller which response to the preprogrammed timer based on real time sensor detection on a certain road junction.

When authorities talk about efficiency and accuracy on real time traffic flow control, there are always room for further enhancement especially on the controlling and sensing method. The new sensing method is capable of counting the total number of vehicles entering a certain junction and exiting from a certain junction on real time basis. Based on this detection, the programmable logic controller will trigger the traffic light indicators according to real demand. 
Traffic management on the road has become a severe problem of today's society because of growth of the urbanization. This leads to traffic jam at the traffic junctions which in Turn causes delay to ambulances. In order to overcome this problem, this paper presents simple ambulance controlled traffic system. The main objective of this system is that to control the traffic, allowing an ambulance to arrive at a particular location without it having to stop anywhere until the destination is reached. This system includes RF Transmitter \& Receiver technology and Lab VIEW software.

\section{OBJECTIVE.}

To develop Traffic Light controller for Congested Urban Route using PLC, this includes Sensing technology, existing similar system and also the suitable programming language that will be used to develop the present sequence method \& to design a prototype of Ambulance Detection using RF Transmitter \& Receiver with Lab View.

\section{OVERVIEW OF PLC}

The PLC is an industrial computer. It is capable of storing instructions to implement control functions such as sequencing, timing, counting, arithmetic, data manipulation and communication. The I/O interfaces provide the connection between the PLC and the information providers (inputs like pushbuttons, sensors) and the controllable devices (outputs like valves, relays, lamps). PLCs are specifically designed to survive the harsh conditions of the industrial environment. A well designed PLC can be placed in an area with substantial amounts of electrical noise, electromagnetic interference, mechanical vibration, and non condensing humidity. The hardware interfaces for connecting field devices are actually part of the PLC itself and are easily connected.

There are different types of Seimens PLC like S7-200, S7-300\& S7-1200 etc.

S7-1214 DC/DC/Relay PLC has been used. S7-1214 DC/DC/Relay has been shown in Figure 1.

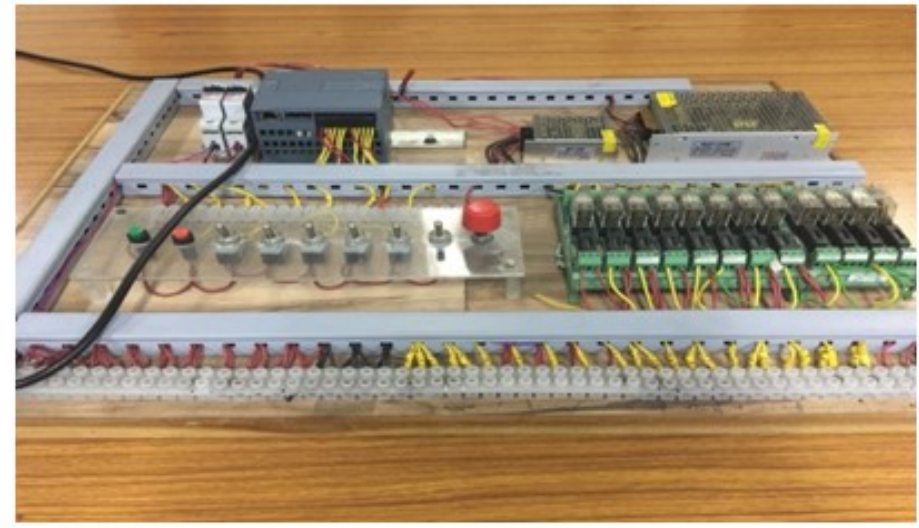

Fig 1: Seimens S7-1214 DC/DC/ RELAY PLC

\section{BLOCK DIAGRAM}

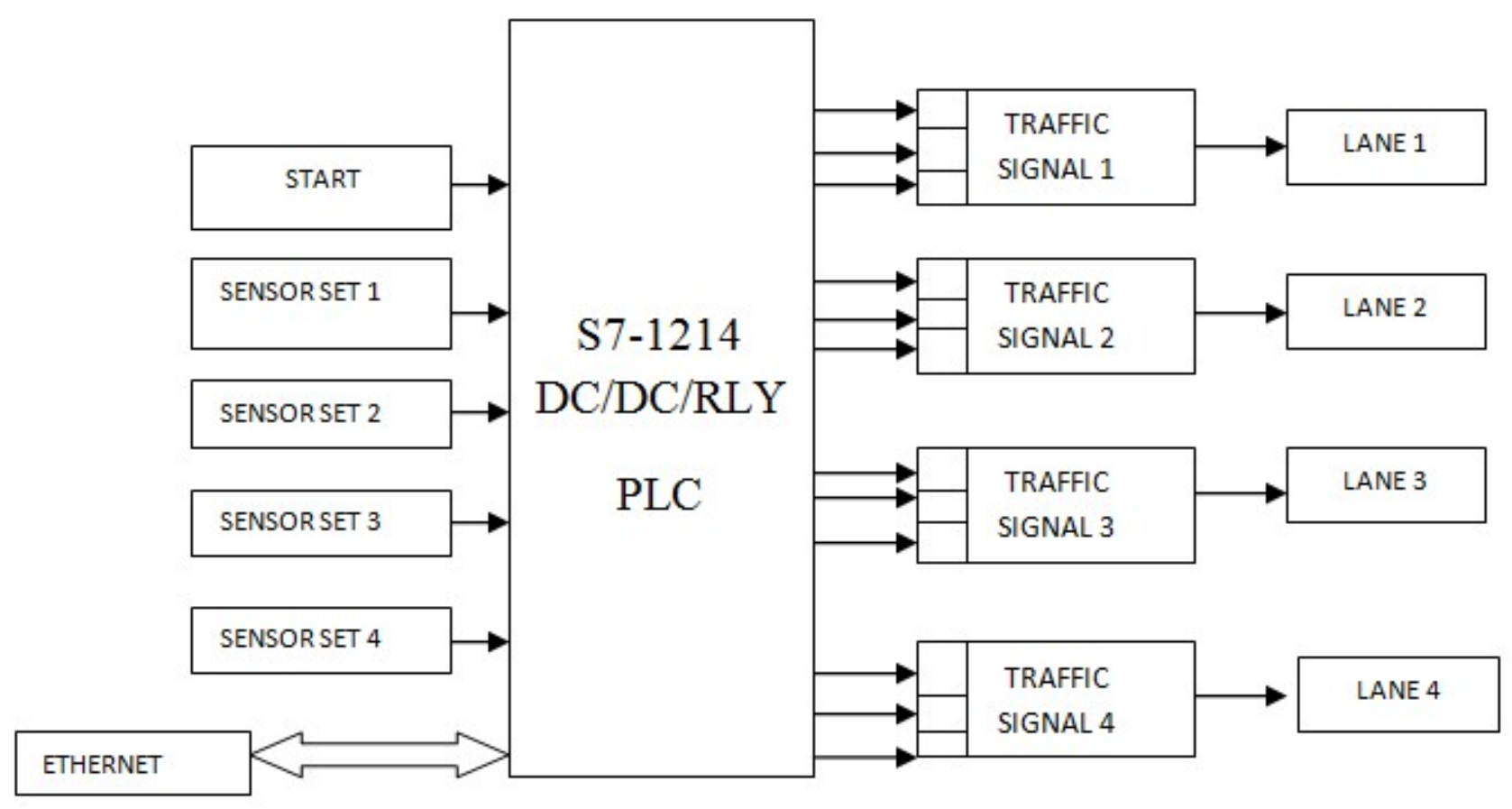

Fig 2: Block diagram of traffic light control using PLC 
The overall setup consists of PLC S7-1214 DC/DC/RELAY, Sensors, and Green, Red, Yellow lights. PLC is connected to the PC through Ethernet, Sensors are connected to PLC, and Power supply is 24V DC.

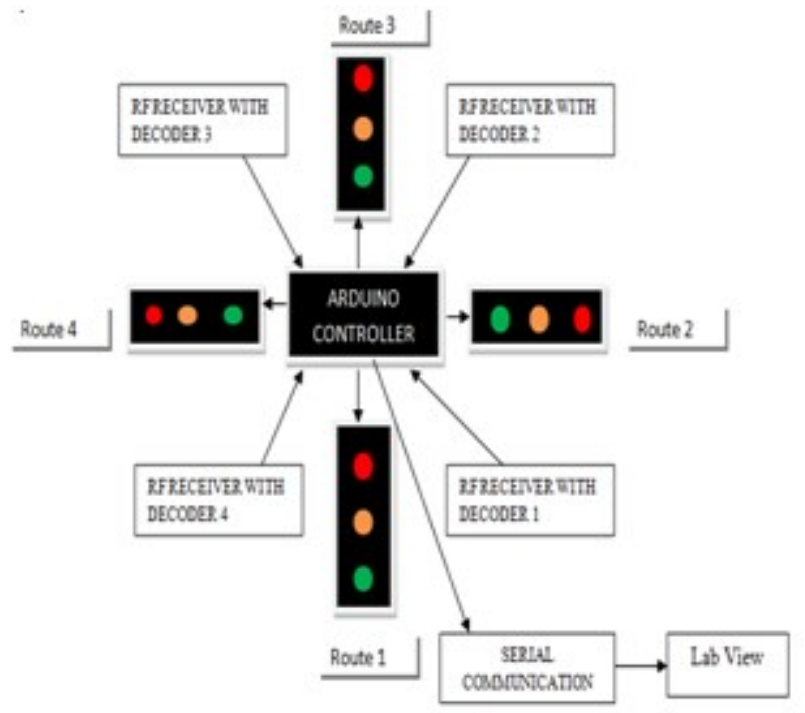

Fig 3: Block diagram of ambulance detection using wireless RF module.

The main objective of this system is that to control the traffic, allowing an ambulance to arrive at a particular location without it having to stop anywhere until the destination is reached. This system includes RF Transmitter \& Receiver technology and Lab VIEW software. An RF Transmitter \& Receiver module reads the respective decoder number from the corresponding ambulance RF transmitter encoder and then it is sent to Arduino controller, which is programmed, with the help of Labview. This controller is capable of communicating with input and output modules. The wireless RF transmitter \& Receiver readers provide the information to the Arduino controller so that it compares the received Signal with default Signal stored in its memory. If the obtained Signals get matched with any of the Signal's, then a green indication is given along the path of the ambulance or else no change in the signal takes place. Figure 3 shows the Block diagram of wireless RF module for Ambulance Detection.

\section{PROPOSED METHODOLOGY}

\section{- Traffic light control for heavily congested urban route using PLC:}

The self-algorithm traffic light system is made up by combination of the sequencing programming method and the sensor based programming method in the PLC as the main controller. The self-Algorithm traffic light system requires additional sensors for better sensing for the vehicles coming in and out of on a certain junction.
The sensors placement for self-algorithm traffic light system is shown in figure 4 .

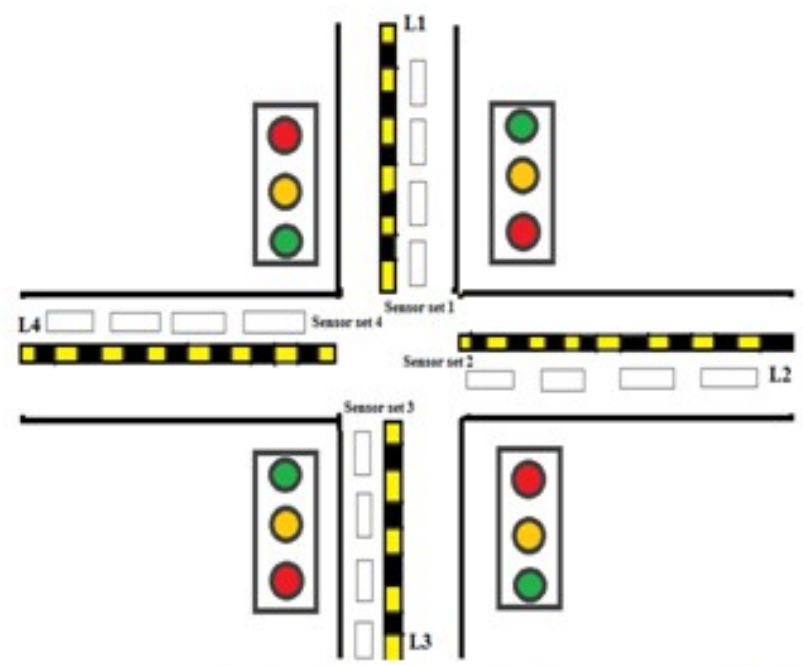

Fig 4: Sensor Placement for self algorithm traffic light system

In each and every lane fours sensors are placed to detect the no of vehicles named as

- $\quad$ SENSOR SET 1: $\mathrm{SL}_{1.0}, \mathrm{SL}_{1.1}, \mathrm{SL}_{1.2} \& \mathrm{SL}_{1.3}$.

- $\quad$ SENSOR SET 2: $\mathrm{SL}_{2.0}, \mathrm{SL}_{2.1}, \mathrm{SL}_{2.2}, \& \mathrm{SL}_{2.3}$

- $\quad$ SENSOR SET 3: $\mathrm{SL}_{3.0}, \mathrm{SL}_{3.1}, \mathrm{SL}_{3.2}, \& \mathrm{SL}_{3.3}$

- SENSOR SET 4: $\mathrm{SL}_{4.0}, \mathrm{SL}_{4.1}, \mathrm{SL}_{4.2}, \& \mathrm{SL}_{4.3}$ 
As simple test bed for the developed prototype of selfalgorithm traffic light system is tested with the new placements of sensor. The first condition is similar to conventional traffic light system which is when the $\mathrm{SL}_{1.0}$ is activated; the timing for the green color traffic light indicator in certain junction will turn on for a period of approximately 5 seconds.

The second condition is when both $\mathrm{SL}_{1.0}$ and $\mathrm{SL}_{1.1}$ is activated; the timing for green color traffic light indicator will be extended for that particular junction for a period of approximately 10 seconds.

The third condition is when both $\mathrm{SL}_{1.0}, \mathrm{SL}_{1.1} \& \mathrm{SL}_{1.2}$ is activated; the timing for green color traffic light indicator will be extended for that particular junction for a period of approximately 15 seconds.

Similarly the fourth condition is when SL1.0, $\mathrm{SL}_{1.1}$, $\mathrm{SL}_{1.2}$ \& $\mathrm{SL}_{1.3}$ is activated; the timing for green color traffic light indicator will be extended for that particular junction for a period of approximately 20seconds.

\section{Ambulance Detection using RF Transmitter \& Receiver with Lab View}

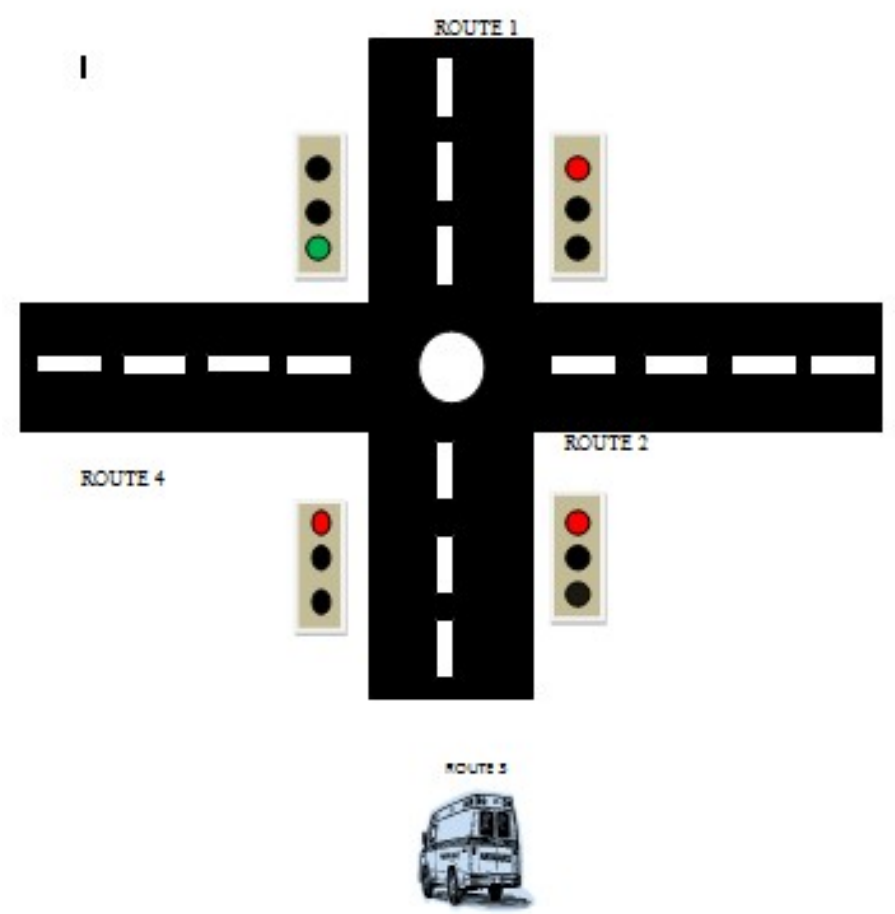

Fig 5: Ambulance detection using RF wireless module.
Firstly, whenever the ambulance arrives at particular junction, the ambulance driver has to show his corresponding lane. RF Transmitter along with an encoder module is fitted into Ambulance, in which over all control is given to Ambulance driver. Then, the reader detects the radio frequency signal and generates corresponding identification code Transmitter \& Receiver module reads the respective decoder number from the corresponding ambulance $\mathrm{RF}$ transmitter encoder and then it is sent to Arduino controller, which is programmed, with the help of Lab view. This controller is capable of communicating with input and output modules. The wireless RF transmitter \& Receiver readers provide the information to the Arduino controller so that it compares the received Signal with default Signal stored in its memory. If the obtained Signals get matched with any of the Signal's, then a green indication is given along the path of the ambulance or else no change in the signal takes place.

\section{SOFTWARE SIMULATION}

\section{PLC SIMULATION FOR DENSITY CONTROL.}

The programming language used is "Ladder Logic". It consists of timers, counters, inputs (switches) and outputs (coils). Below simulation figures shows the traffic controller ladder logic. The plc code is downloaded and tested. 
International Journal of Trend in Scientific Research and Development (IJTSRD) ISSN: 2456-6470
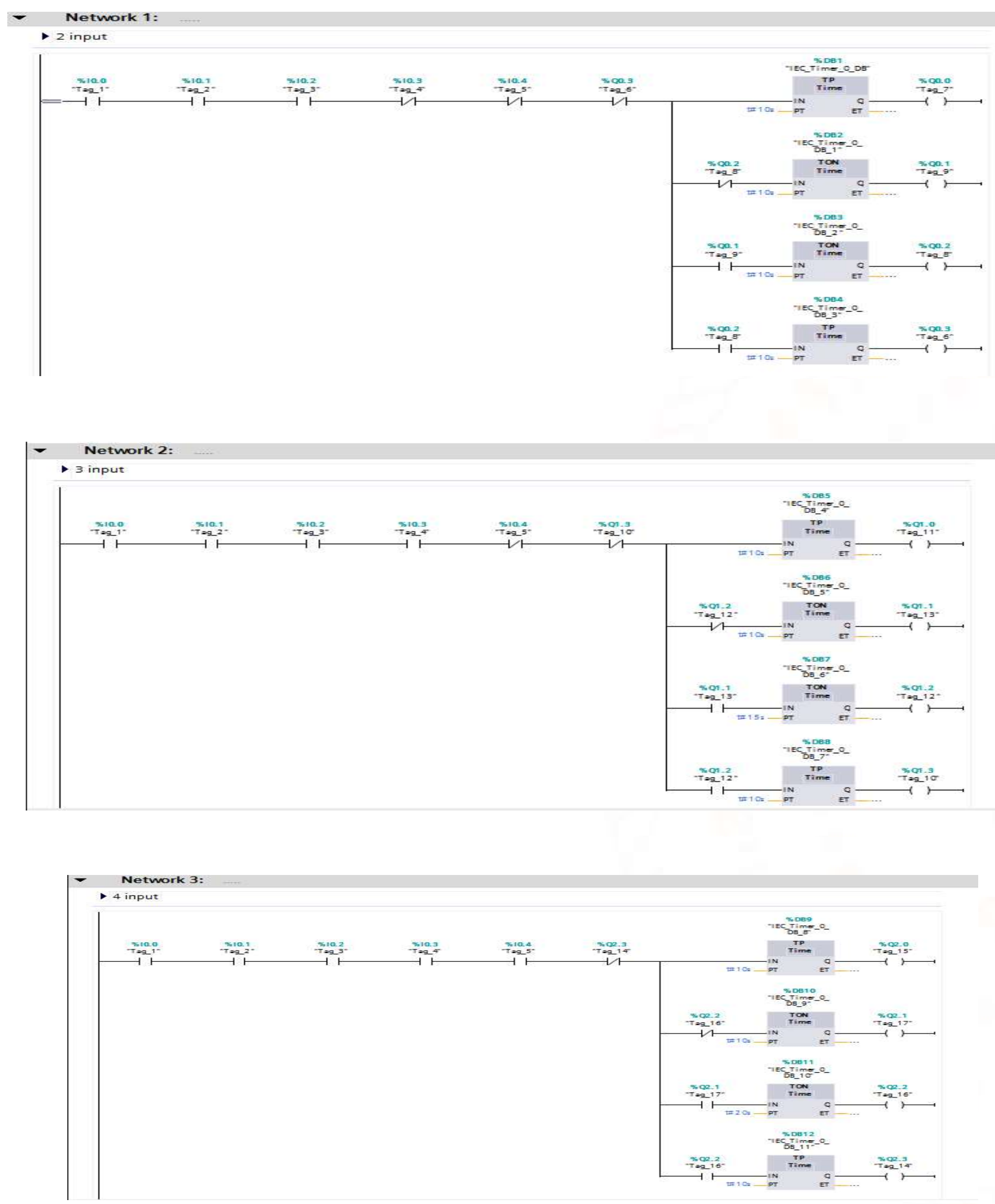

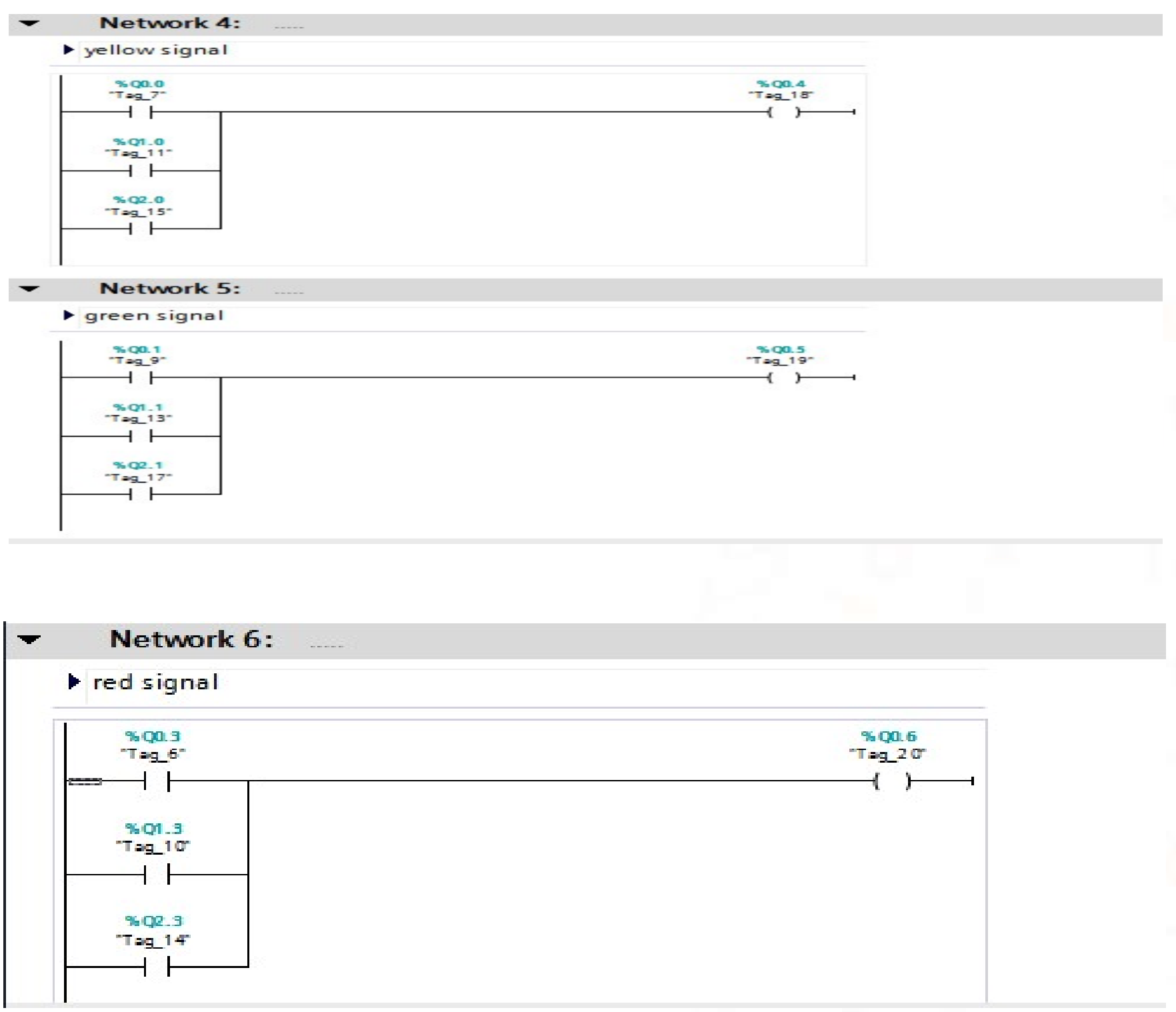

Fig 6: PLC ladder diagram for traffic light control

\section{$>$ AMBULANCE DETECTION USING LAB VIEW}

This system performs as a normal traffic system in the absence of ambulance. The operation gets altered whenever the ambulance arrives at a particular junction. The performance will be observed on the front panel of the Lab VIEW. The Block diagram of LabVIEW are as follows respectively.

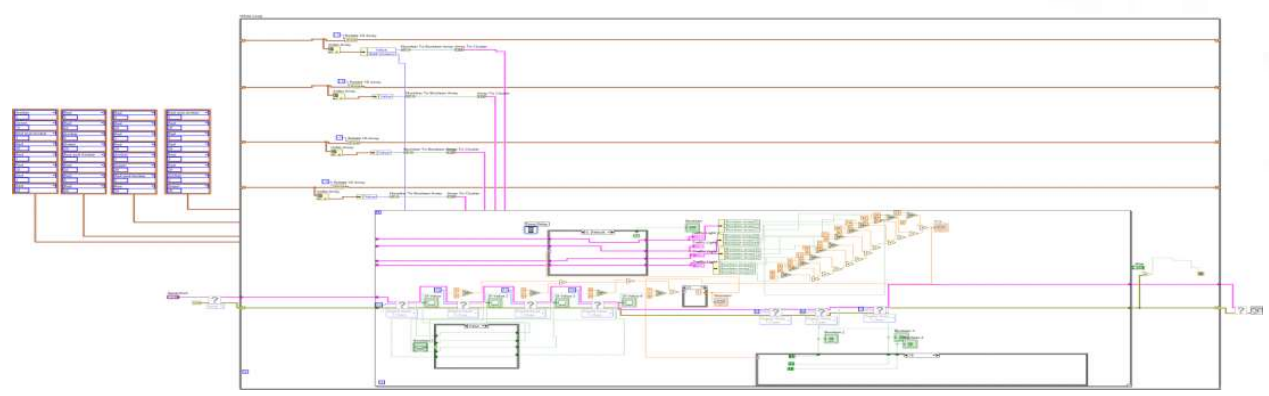

Fig 7: Ambulance detection simulation using Lab View. 


\section{CONCLUSION}

An intelligent traffic light system had successfully been designed and developed. The sensors were interfaced with S7-1200 PLC module. This interface is synchronized with the whole system of the traffic system. This project can easily be implemented in real life situation. Increasing the no. of sensors to detect the presence of vehicles can further enhance the design of the traffic light system. Another room of implement is to have the photoelectric sensors replaced with an imaging system, camera system so that it has a Wide range of detection capabilities which can be enhanced and ventured into a perfect traffic system.

Ambulance detection system will definitely help the ambulance by giving the way to it when there is a heavy traffic on the road. This system uses the Lab VIEW software to monitor the traffic whenever ambulance arrives at the junction. The operation which is performed in the hardware circuit is similarly observed on the front panel of Lab VIEW. The designed system has simple architecture, fast response time, ease in understanding the working module, user friendliness and scope for further expansion.

\section{FUTURE SCOPE.}

In future, pedestrian control logic can be added with this fuzzy traffic controller. The entire traffic controller in the city can be connected by creating network connection and can communicate with each other with sending information about current traffic situation of their intersections, which will be using as inputs for the fuzzy controller. On the basis of these inputs the controller will produce output to better control of the congestion considering the current traffic situations of other neighbor traffic controller's intersection of the network.

Ambulance control system can be extended by introducing GSM module, which sends alert intimation to the respected hospital authorities stating that ambulance is near to the hospital. In future we can use this system in several applications by adding additional components to it.

\section{REFERENCES}

1. Muhammad ArshadKhattak "PLC Based Intelligent Traffic Control System", International Journal of Electrical \& Computer Sciences IJECSIJENS Vol: 11 No: 06 @ December 2011.

2. Carlo Y. Makdisie, "Automation Development of Traffic Light Control via PLC based Simatic Manager", International Journal of Electronics Engineering, 4 (1), 2012, pp. 85- 88.

3. S. K. Subramaniam, V. R. Gannapathy, S. A.Anas, A. B. M. Diah, M. K. Suaidi, and A. H.Hamidon, Intelligent and Self Control SafetyTraffic Light System for Road Constructions,Proceedings of 8th WSEAS International Conference on Circuits, Systems, Electronics,Control and Signal Processing, December 14-16, 2009, pp. 53-58.

4. Pappis, C. P., and E. H. Mamdani, A Fuzzy Logic Controller for a Traffic Junction, IEEE Transactions Systems, Man, and Cybernetics, Vol. SMC-7, No. 10, October 1977,pp.707 717.

5. MohitDevSrivastava, Prerna, Shubhendu Sachin3, Sumedha Sharma4, UtkarshTyagi "Smart traffic control system using PLC and SCADA" ijirset Vol. 1, Issue 2, December 2012.

6. C. M. Mwangi, S. M. Kang'ethe and G. N. Nyakoe "Design and simulation of a fuzzy logic traffic signal controller for a signalized intersection". 